\title{
The Application of Regression Analysis in Modeling Interconnect Resistance
}

\author{
Chen Baojun \\ School of Electronics and Information Engineering \\ DaLian JiaoTong University \\ Dalian, China \\ beec98@163.com
}

\author{
Ju Yanjie \\ School of Electronics and Information Engineering \\ DaLian JiaoTong University \\ Dalian, China \\ yanjieju@sina.com
}

\author{
Zhao Ke \\ School of Electronics and Information Engineering \\ DaLian JiaoTong University \\ Dalian, China \\ Zhaoke2006@hotmail.com
}

\begin{abstract}
As clock frequencies exceed giga-Hertz, the skin depth in analog and digital circuits decreases heavily. The technology scaling have lead to complications that pose significant challenges to on-chip interconnect design, such as the distortion of signal pulses. One of the most important causes of pulse distortion is the frequency-dependence of conductor loss, which can be incorporated into circuit models for interconnects as frequency-dependent resistance per-unit-length. This makes accurate resistance necessary in simulation. In order to achieve high simulation efficiency, closed-form formulae are often used to represent frequencydependent resistance of interconnects. In this letter, two closed-form formulae for the frequency-dependent per-unitlength resistance of rectangular cross-sectional interconnects are presented. The frequency-dependent per-unit-length resistance of a rectangular interconnect line or an interconnect line with a ground plane structure is first obtained by the method of moments (MOM). Based on this strict numerical MOM results, the novel closed-form formulae for a rectangular interconnect are fitted out using the regression analysis. The formulae can be widely used for analyzing on-chip power grid IR-drop when the frequency is changing. Compared to the previously published formulae for an interconnect, the formula provided here is more accurate during the frequency transition range.
\end{abstract}

Keywords- interconnect; resistance;Regression analysis;MOM;IC

\section{INTRODUCTION}

In the multi-gigahertz frequency range, skin effect causes current distributing to flow only at the surface of conductors, leading to a decrease of wire inductance and an increase of resistance. The latter effect causes a signal attenuation which increases with frequency. Many computed-aided design tools for microwave circuits use field simulation and analysis techniques based on frequency-domain or time domain electromagnetic (EM) numerical methods to compute the ac resistance ${ }^{[1-5]}$. However, these methods require intensive computations in central processing unit (CPU) and make device optimization a formidable task.

In order to reduce the cost of needed time, some software use simple formulas to compute the ac resistance, such as :

$$
r(f)=\sqrt{r_{d c}^{2}+r_{h f}^{2}}
$$

where $r_{d c}=1 / \sigma w t, r_{h f}=\frac{1}{2(w+t)} \sqrt{\frac{\pi f \mu}{\sigma}}$

$r_{d c}$ is the direct resistance, $\sigma$ is the conductivity of the conductor, $w$ and $t$ are the width and the thickness of the rectangular cross-section.

In 2000, Chen ${ }^{[6]}$ published empirical closed formulas(2) for single conductor of rectangular crosssection.

$$
\begin{gathered}
r(f)=r_{d c}\left[a\left(\frac{f}{f_{0}}\right)^{p}+1\right] \quad f<f_{0} \\
r(f)=r_{d c}\left[a\left(\frac{f}{f_{0}}\right)^{-q}+\sqrt{\frac{f}{f_{0}}}\right] \quad f \geq f_{0}
\end{gathered}
$$

Where $f_{0}=\frac{4}{\pi \mu_{0} \sigma}\left(\frac{w+t}{w t}\right)^{2}$. 
The results of the published formulas are acceptable within limited range. For example, the ac resistance of wires with high aspect radios (width-to-thick) is much smaller than the results of conventional numerical method. Another problem of the existing formulas is that none of them considers proximity effect induced by plane, so the application of them is confined.

In this letter, two suitable formulas of rectangular conductor resistance exploiting regression are proposed. These formulas combine high accuracy, large range of geometrical and reduced CPU time. Also, we compare the results with the conventional numerical method.

\section{REGRESSION ANALYSIS}

Regression analysis is a conceptual simple method for investigating function relationships among variables. The is expressed in the form of an equation or a model connecting the response or dependent variable and one or more explanatory or predictor variables ${ }^{[7]}$. A general regression equation is:

$$
y=u(x)+\varepsilon
$$

where $y$ is the response, variable $x$ is the predictor variable, $u(x)$ is the fitting function and $\varepsilon$ is the random disturbance or residual.

$R^{2}$ (Determinants of coefficient) measures how much of the variation in the simulation response is explained by the regression model. If $R^{2}$ is near 1 , then the fitted value accounts for a large part of the variation in the mean value.

$$
y=\beta_{0}+\beta_{1} x_{1}+\beta_{2} x_{2}+\cdots \beta_{p} x_{p}+\varepsilon
$$

Equation (4) is the equation of multiple linear regression .It described the relationship between response variable $y$ and $p$ predictor variables $x_{1}, x_{2}, \ldots x_{p}$. $\beta_{0}, \beta_{1}, \beta_{2}, \cdots \beta_{p}$ are constants referred to as the model partial regression coefficients.

Besides $R^{2}$, Durbin-Watson statistic is also the basis of a popular test of autocorrelation in multiple linear regression analysis. The closer the value of $\mathrm{d}$ to 2 , the firmer the evidence that there is no autocorrelation present in the residuals.

\section{THE CLOSED-FORM FORMULAE}

In 1918, Dwight $^{[8]}$ introduced the principle of similitude which states that the ratio of ac to dc resistances for an isolated rectangular strip conductor is a function of two variables, namely, the ratio of strip width to thickness $(w / t)$, and the frequency. The function is dependent on the material surrounding the strip and the presence of a ground.

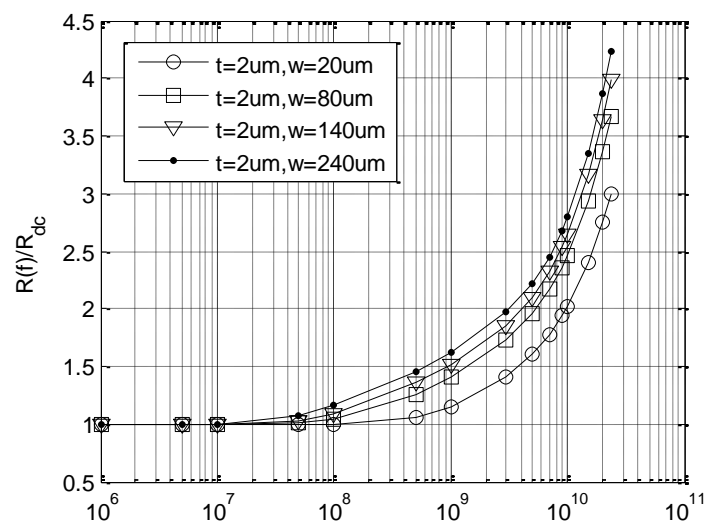

Figure 1. Comparison of the computed $\mathrm{r}(\mathrm{f}) / \mathrm{rdc}$ of single conductor with numerical method.

Fig. 1 presents the frequency-dependent resistance obtained by the numerical method for single rectangular line. In the picture we do the normalization which means the picture shows $R(f) / R_{d c}$ instead of $R(f)$. For different $w / t$ (width to thickness) ratios, we can see all the $R(f)$ convergent to the $R_{d c}$ at low frequency. As frequency is increased, wider line first shows skin effects while fine line shows the skin effect later.

By analyzing we conclude that within the range of high frequency the relation of $\ln r(f)$ and $\ln \left(f / f_{0}\right)$ can be described by a quadratic function, so the formula we propose here, the frequency-dependent resistance is:

$$
\begin{aligned}
& r_{h}(f)=e^{C+B_{1} \ln \frac{f}{f_{0}}+B_{2}\left(\ln \frac{f}{f_{0}}\right)^{2}} f<f_{b} \\
& r_{l}(f)=k+n f+m f^{2} \quad f \geq f_{b}
\end{aligned}
$$

Formula (4a) describes the ac resistance when the frequency is high and (5b) corresponds to the low frequency. In formula (5a) the parameters $C, B_{1}, B_{2}$ correspond to the dimension of the conductor. Using multiple linear regression the parameter $\mathrm{C}$ can be described by function (6), similarly receiving function (7), (8), (9).

$$
\begin{gathered}
C=0.468+0.974 \ln r_{d c}+0.09 \ln \frac{w}{t} \\
B_{1}=0.375-0.021 \ln \frac{w}{t}+5 \times 10^{7} \cdot w \cdot t \\
B_{2}=0.048-3653.417 \cdot t \\
f_{b}=4 \times 10^{7}+2297833 \cdot r_{d c}+1300194 \cdot \frac{w}{t}
\end{gathered}
$$

In $(5 b)$ to maintain the continuity the first derivation of $r(f)$ at $f_{b}$, we found that: $R_{l}(0)=R_{d c}, R_{l}\left(f_{b}\right)=R_{h}\left(f_{b}\right), R_{l}\left(f_{b}\right)^{\prime}=R_{h}\left(f_{b}\right)^{\prime}$. So we receive the values of $k, m$ and $n$.

Data in Tab.1 can show the formula of the four parameters is valid. 
TABLE I. THE RESULTS OF MULTIPLE LINEAR REGRESSION

\begin{tabular}{|c|c|c|}
\hline parameter & $R^{2}$ & $d$ \\
\hline$C$ & 0.999 & 1.872 \\
\hline$B_{1}$ & 0.902 & 1.923 \\
\hline$B_{2}$ & 0.913 & 1.89 \\
\hline$f_{b}$ & 0.921 & 1.904 \\
\hline
\end{tabular}

The ac resistance of conductor influenced by plane is different with that of single conductor. Because of the proximity effect the current distribution facing plane in the conductor is much larger, so the ac resistance increases.

Fig.2 shows the $R(f) / R_{d c}$ for a single line with a ground plane nearby (where $d$ is the distance between the center of the line and the ground place surface). From the picture, one can see that as the ground plane introduction, the $R(f)$ start increase at early frequency. Also if the ground plane is closer, the proximity effect is heavier. (the line dimension used in fig. $5 \mathrm{~b}$ is $w=6 u m, t=4 u m$ ).

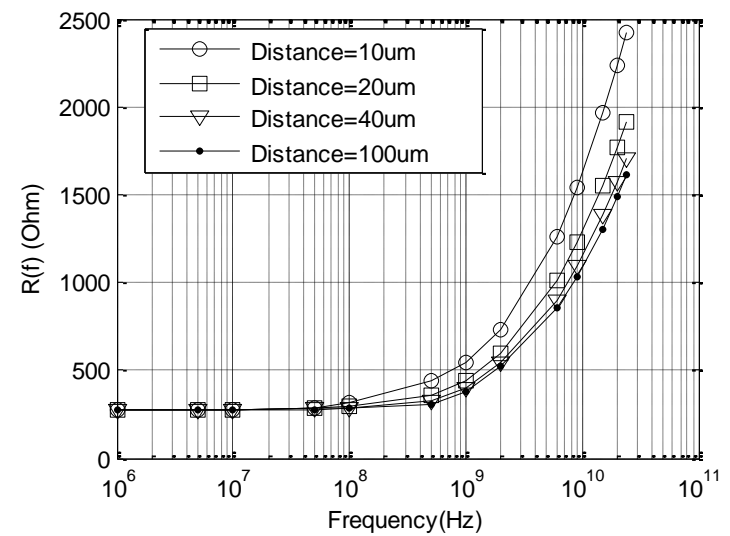

Figure 2. Comparison of the computed $\mathrm{r}(\mathrm{f}) / \mathrm{rdc}$ of conductor with numerical method.

The proximity effect relates with the distance between conductor and plane. Therefore the ac resistance is changing with the distance. The operating frequency, $f$, has been given in terms of a more useful parameter: $p=\sqrt{2 \mu \sigma f A}$ known as the normalized frequency in the literature ${ }^{[9]}$, where $A$ is the cross-section area of the conductor. So the second model is flowing:

$$
\begin{array}{ll}
r_{h}(f)=r_{d c}\left(C+B_{1} \ln p+B_{2} \ln p^{2}\right) f<f_{b} \\
r_{l}(f)=k+n f+m f^{2} \quad f \geq f_{b}
\end{array}
$$

Similar to the first model the parameters $C, B_{1}, B_{2}$ in function (10a) correspond to the distance and dimension of the conductor.Fig. 3 represent the parameter $C, B 1, B 2$ with different distance. According to the figures we can have the flowing functions(11-13):

$$
\begin{aligned}
& C=e^{b_{01}+\frac{b_{02}}{d i s}} \\
& B_{1}=-e^{b_{11}+\frac{b_{12}}{d i s}} \\
& B_{2}=e^{b_{21}+\frac{b_{22}}{d i s}}
\end{aligned}
$$

$$
\begin{aligned}
& \text { Where } b_{01}=-0.119+204.304 \cdot \frac{1}{\mathrm{R}_{\mathrm{DC}}}, \\
& b_{02}=\left(0.582+1104.015 \cdot \frac{1}{\mathrm{R}_{\mathrm{DC}}}\right) \times 10^{-6}, \\
& b_{11}=-0.884+419.6024 \cdot \frac{1}{\mathrm{R}_{\mathrm{DC}}} \\
& b_{12}=\left(1.584+1003.585 \cdot \frac{1}{\mathrm{R}_{\mathrm{DC}}}\right) \times 10^{-6} \\
& b_{21}=-0.442+166.167 \cdot \frac{1}{\mathrm{R}_{\mathrm{DC}}} \\
& b_{22}=\left(1.827+863.608 \cdot \frac{1}{\mathrm{R}_{\mathrm{DC}}}\right) \times 10^{-6}
\end{aligned}
$$

Data in Tab.2 shows the formula of the parameters is valid.

TABLE II. THE RESULTS OF MULTIPLE LINEAR REGRESSION

\begin{tabular}{|c|c|c|c|}
\hline parameter & $C$ & $B_{1}$ & $B_{2}$ \\
\hline$R^{2}$ & 0.999 & 0.999 & 0.998 \\
\hline parameter & $b_{01}$ & $b_{02}$ & $b_{11}$ \\
\hline$R^{2}$ & 0.975 & 0.987 & 0.979 \\
\hline parameter & $b_{12}$ & $b_{21}$ & $b_{22}$ \\
\hline$R^{2}$ & 0.981 & 0.974 & 0.982 \\
\hline
\end{tabular}

\section{RESULTS}

Formula (5) is an explicit expression which contains the frequency and the line size as variables. To validate the formula, we compare it with the numerical results and Chen formula ${ }^{[4]}$.

Fig.3 shows the comparison between the different resistance values of a single line: the proposed formula (5) results, Chen formula ${ }^{[6]}$ results and the numerical results. The numerical results are regarded as the standard true results. From the picture, one can see our proposed formula results are always agree well with numerical results in all frequency range and with different dimension sizes. The maxim relative error is less than $5 \%$. While for Chen formula ${ }^{[4]}$, for 20 um width and 2 um thickness line, it matches well with the numerical results (figure 3.a) with about $10 \%$ error in the transition frequency range; but in case of 200um width and 2um thickness line, Chen's formula results are far away from numerical results which show more than $50 \%$ error in figure 3.b. These experiments show our formula (5) is valid in larger line geometry range and is much better than Chen's formula.

Balachandran ${ }^{[10]}$ derived a formula to calculate the resistance with taking ground plane into account. Figure 4 compares the frequency-dependent resistances considering the proximity effect with the formula (10) proposed in this paper and Balachandran formula ${ }^{[5]}$. It can be seen that when the distance between conductor and ground changes, the formula(10) agree fairly well with that from numerical computation. Also figure 4 shows our formula's accuracy advantages over the Balachandran formula in large line $w / t$ ratio ranges. 
Compare the frequency-dependent resistances computed from conventional numerical method with formula (5) and (10) proposed in this letter. From Fig.3 and Fig. 4 it can be seen that the results of two formulas are approximate.

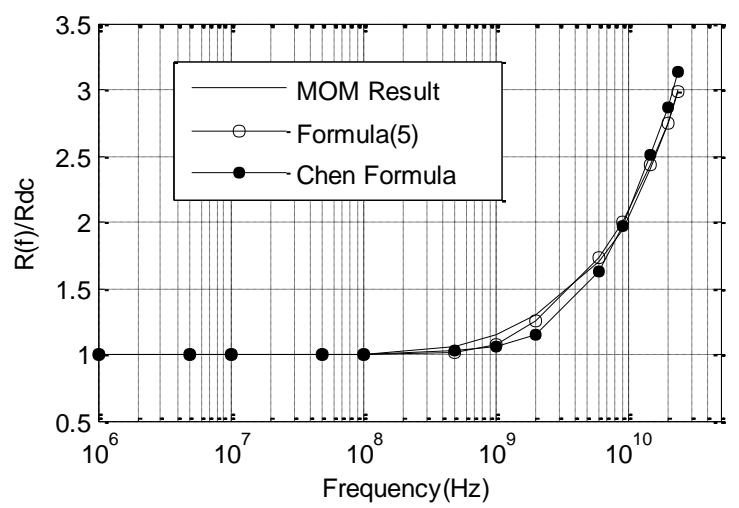

(a)

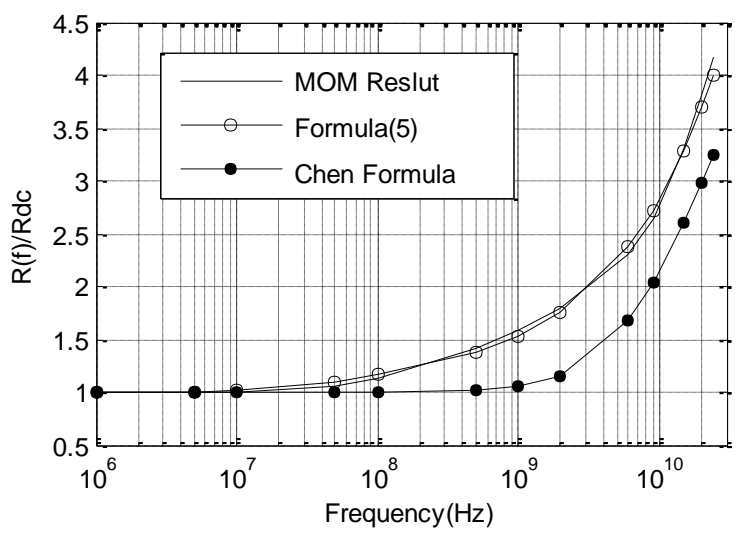

(b)

Figure 3. Comparison of the results computed $r(f)$ by formula (5) with numerical method. In (a), line size is $20 \mathrm{um}^{*} 2 \mathrm{um}$; in (b), line size is 200um*2um.

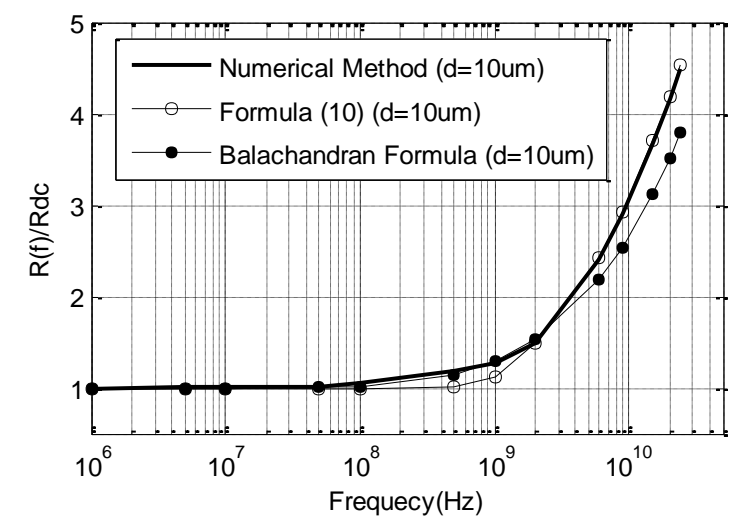

(a)

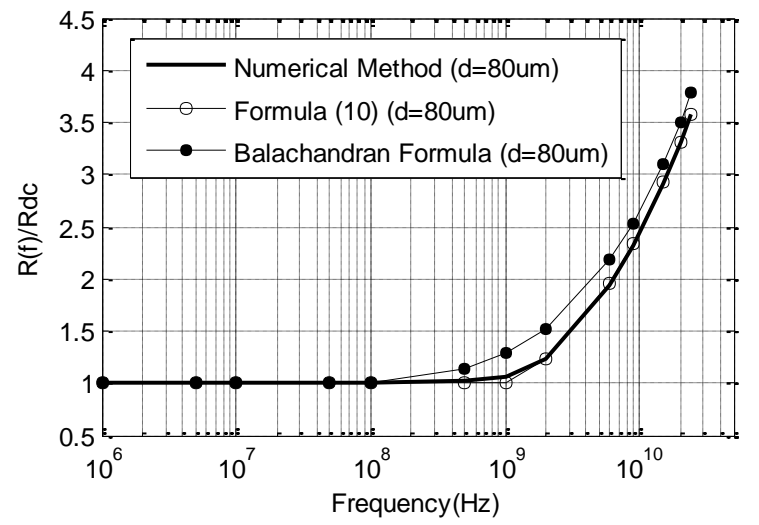

(b)

Figure 4. Comparison of the results computed $r(f)$ by formula (10) with numerical method.(d represents distance)

\section{CONCLUSION}

In summery, we have obtained new formulas for frequency-dependent resistance of rectangular crosssection conductors. Formula (5) can be used to compute ac resistance of single conductor with large range of geometrical and formula (10) is the first one to compute ac resistance considering the proximity effect. When using the formulas, the CPU time consumption can be reduced significantly.

\section{REFERENCES}

[1] B. Mukherjee, W. Lei , and A. Pacelli , "A practical approach to modeling skin effect in on-chip interconnects," Proceedings of the 14th ACM Great Lakes symposium on VLSI,pp.266-270,2004.

[2] D. Hinojosa, I.Domenech, O.Martinez, and I.Garrigos, "Empirical model generation techniques for planar microwave components using electromagnetic linear regression models,",Microwave Symposium Digest, 2005 IEEE MTT-S International,pp.1143-1146, June 2005.

[3] J. Michael, and J. A. Kong. "A Hybrid Method for the Calculation of the Resistance and Inductance of Transmission Lines with Arbitrary Cross Sections," Microwave Theory and Techniques, vol.39, pp.1338-1347, Aguest 1991.

[4] Z. Wang, and W. Qiming. "A two-dimensional resistance simulator using the boundary element method". Computer-Aided Design of Integrated Circuits and Systems, vol.11, pp. 497-504, April 1992.

[5] F. Dana, and R.Chow, "Edge condition of the field and a.c. resistance of a rectangular strip conductor," Microwaves, Antennas and Propagation, vol.137,,pp. 133-140,April. 1990.

[6] C. Huabo, and J. yuan fang,"Modeling of impedance of rectangular cross-section conductors," Electrical Performance of Electronic Packaging, IEEE Conference on,pp.159-162,October 2000.

[7] C. Chatterjee, and D. Samprit, "Regression Analysis by Example,"pp.1-3,2002.

[8] H. B. Dwight, "Skin effect in tabular and flat conductor," American Institute of Electrical Engineers, Transactions , vol 25, pp. 1379-1403,July 1918.

[9] P. Waldow and I. Wolff, "The skin effect at high frequency," IEEE Trans. Microwave Theory Tech., vol. MTT-33, pp. 10761082,Oct,1985.

[10] J. Balachandran , S. Brebels, and G. Carchon, "Compact broadband resistance model for microstrip transmission lines," Electrical Performance of Electronic Packaging, vol.45, pp.84-87, 2004. 